\title{
Verbal and Visual Memory Impairments in Bipolar I and II Disorder
}

\author{
Tae Hyon Ha'1, Ji Sun Kim¹, Jae Seung Chang ${ }^{1}$, Sung Hee Oh${ }^{1}$, Ju Young Her', \\ Hyun Sang $\mathrm{Cho}^{2}$, Tae Sung Park ${ }^{3}$, Soon Young Shin ${ }^{4}$ and Kyooseob $\mathrm{Ha}^{1,5} \bowtie$ \\ ${ }^{1}$ Mood Disorders Clinic \& Clinical Affective Neuroscience Laboratory, Department of Psychiatry, Seoul National University Bundang Hospital, \\ Seongnam, Republic of Korea \\ ${ }^{2}$ Department of Psychiatry, Severance Mental Health Hospital, Gwangju, Republic of Korea \\ ${ }^{3}$ Department of Statistics, Seoul National University, Seoul, Republic of Korea \\ ${ }^{4}$ Institute of Biomedical Science and Technology, Konkuk University Hospital, Seoul, Republic of Korea \\ ${ }^{5}$ Department of Psychiatry, Seoul National University College of Medicine and Institute for Human Behavioral Medicine, Seoul, Republic of Korea
}

Objective To compare verbal and visual memory performances between patients with bipolar I disorder (BD I) and patients with bipolar II disorder (BD II) and to determine whether memory deficits were mediated by impaired organizational strategies.

Methods Performances on the Korean-California Verbal Learning Test (K-CVLT) and the Rey-Osterrieth Complex Figure Test (ROCF) in 37 patients with BD I, 46 patients with BD II and 42 healthy subjects were compared. Mediating effects of impaired organization strategies on poor delayed recall was tested by comparing direct and mediated models using multiple regression analysis.

Results Both patients groups recalled fewer words and figure components and showed lower Semantic Clustering compared to controls. Verbal memory impairment was partly mediated by difficulties in Semantic Clustering in both subtypes, whereas the mediating effect of Organization deficit on the visual memory impairment was present only in BD I. In all mediated models, group differences in delayed recall remained significant.

Conclusion Our findings suggest that memory impairment may be one of the fundamental cognitive deficits in bipolar disorders and that executive dysfunctions can exert an additional influence on memory impairments.

Psychiatry Investig 2012;9:339-346

Key Words Bipolar I disorder, Bipolar II disorder, Memory, Organization, Executive function, Path analysis.

\section{INTRODUCTION}

An increasing number of studies have detected cognitive impairments in patients with bipolar disorders not only during acute phase but also during euthymic periods. ${ }^{1}$ Broad cognitive impairments were present even in early phase of illness ${ }^{2}$ and in unaffected relatives ${ }^{3}$ as well, suggesting that certain domains of cognitive deficits may be a potential endophenotype. According to a recent meta-analysis, ${ }^{1}$ the largest effect sizes were noted for executive functions and verbal memory. How-

Received: September 16, 2012 Revised: October 7, 2012

Accepted: October 7, 2012 Available online: November 12, 2012

$\triangle$ Correspondence: Kyooseob $\mathrm{Ha}, \mathrm{MD}, \mathrm{PhD}$

Department of Psychiatry, Seoul National University Bundang Hospital, 82 Gumi-ro 173beon-gil, Bundang-gu, Seongnam 463-707, Republic of Korea

Tel: +82-31-787-7431, Fax: +82-31-787-4058, E-mail: kyooha@snu.ac.kr

(a) This is an Open Access article distributed under the terms of the Creative Commons Attribution Non-Commercial License (http://creativecommons.org/licenses/by$\mathrm{nc} / 3.0$ ) which permits unrestricted non-commercial use, distribution, and reproduction in any medium, provided the original work is properly cited. ever, it is still unclear whether these impairments reflect multiple independent cognitive deficits or a single fundamental cognitive deficit.

Performances on formal memory tests can be affected by executive functions. Ability to cluster semantically related words into a category or to organize visual information into a meaningful perceptual unit during learning enhances its subsequent recall from memory. For example, patients with fronto-striatal pathology, such as Huntington's disease and Parkinson's disease, are characterized by deficits in free recall that are not related to deficits in storing information but associated to deficits in the organization of information to be remembered. ${ }^{4}$ Likewise, psychiatric patients with obsessive-compulsive disorder $(\mathrm{OCD})^{5-8}$ or schizophrenia, ${ }^{9}$ that are known to be related to fronto-striatal dysfunction as well, have shown deficits in organizational strategies causing impaired memory performances. In bipolar disorders, there is inconsistent evidence for fronto-striatal dysfunction. These circuits seem to be spared 
in volumetric studies. ${ }^{10}$ However, contrarily to volumetric findings, the ventrolateral prefrontal and striatal regions showed abnormal activation in functional studies. ${ }^{11}$ Therefore, investigating the effect of organizational strategy on recall could provide important insights into the pathophysiology of bipolar disorders.

In previous studies, deficits in verbal memory in bipolar disorder were not associated to semantic clustering ${ }^{12}$ and were mediated to a lesser extent by organizational strategies compared to $\mathrm{OCD},{ }^{7}$ suggesting a primary deficit in encoding of verbal information. On the other hand, deficits in visual memory in patients with BD I were mediated by poor use of organizational strategies. ${ }^{13}$ A paucity of research on these relationships limits generalization of findings. Furthermore, these studies investigated patients with $\mathrm{BD} \mathrm{I}^{7,13}$ or a mixed sample of patients with BD I or bipolar II disorder (BD II). ${ }^{12}$ To our knowledge, there has been no study to investigate the mediating effect of strategies on memory performances in BD II.

The clinical course of BD II is distinct from that of BD I. ${ }^{14-16}$ Genetic and neuroimaging findings also suggest differences between BD I and BD II. ${ }^{17-20}$ Regarding cognitive functions of patients with BD II, there is inconsistent evidence for verbal memory and executive functions. ${ }^{21-24}$ In our previous volumetric and diffusion tensor imaging studies, ${ }^{19,20}$ structural brain abnormalities in anterior limbic circuits were common in both subtypes of bipolar disorder, while BD I was characterized by further involvement of the frontal and temporal regions. Therefore, cognitive deficits in BD I would be greater or broader than those in BD II. In accordance with this hypothesis, we found that semantic organization in BD I was poorer than that in BD II. ${ }^{25}$

The purpose of the current study was to compare verbal and visual memory performances between BD I and BD II and to determine whether, if present, memory deficits in BD I and BD II were mediated by organizational strategies. Based on our previous findings, we hypothesized that memory deficits would be greater in BD I than in BD II and that the mediating effect of strategies on memory deficits would be evident in BD I.

\section{METHODS}

\section{Subjects}

The sample consisted of 37 patients with BD I, 46 patients with BD II and 42 healthy subjects. Patients who met the DSMIV criteria for BD I or BD II were recruited from the outpatient clinic or inpatients unit of the Mood Disorders Clinic at Seoul National University Bundang Hospital. Their diagnoses were assessed by using the Structured Clinical Interview for DSM-IV axis I Disorders (SCID) ${ }^{26}$ or the Mini-International Neuropsychiatric Interview (MINI) ${ }^{27}$ Inclusion criteria were absence of any lifetime history of significant neurological or medical illnesses, age between 18 and 60, and estimated IQ over 80 . Patients being in a severe mood episode, which was noted by the treating clinician, were excluded. Clinical states were stratified according to the diagnostic interview or Clinical Global Impression for Bipolar Disorder (CGI-BP $)^{28}$ score at the time of cognitive test. Healthy subjects were recruited by leaflet advertisement. Absence of any axis I psychiatric disorder in the control group was confirmed with the SCID non-patient version. There were no significant differences in age, gender ratio and education years between the three groups (Table 1). However, the mean of estimated IQ was lower in both patients groups than in the control group $(\mathrm{F}=16.887, \mathrm{p}<0.001$ for $\mathrm{BD}$ I and $\mathrm{F}=8.472, \mathrm{p}=0.01$ for BD II group).

The two patients groups did not differ in age at onset, duration of illness, severity of mood symptoms measured by CGIBP, and current mood states. Ten patients with BD I (27.0\%) and 19 patients with BD II (41.3\%) had one or more lifetime or current comorbid psychiatric disorders. However, the proportions of patients with comorbidity did not differ between the patients groups (Table 1). All patients except two with BD II were taking medications. More patients with BD I $(n=26$, $70.3 \%)$ were taking lithium than patients with BD II $(n=22$, $47.8 \%)\left(\chi^{2}=4.236, \mathrm{p}=0.33\right)$. Twenty patients with $\mathrm{BD} \mathrm{I}(54.1 \%)$ and 20 patients with BD II (43.5\%) were taking divalproex. Seven patients with BD I (18.9\%) and 9 patients with BD II (19.6\%) were taking lamotrigine. The proportions of patients who were taking divalproex or lamotrigine were not different between the two patients groups. The daily mean doses of lithium, divalproex and lamotrigine were not different between the groups. Antipsychotics were taken more frequently in patients with BD I $(n=34,91.9 \%)$ than in patients BD II $(n=24$, $69.9 \%)\left(\chi^{2}=15.369, \mathrm{p}<0.001\right)$. Besides, 4 patients with BD II were taking antidepressants, 14 patients with $\mathrm{BD}$ I (37.8\%) and 18 patients with BD II (39.1\%) benzodiazepines, and 2 patients in each group were taking stimulants. This study was approved by the institutional review board and a written informed consent was obtained from all subjects.

\section{Procedures}

The Korean-California Verbal Learning Test (K-CVLT) ${ }^{29}$ was administered to study subjects. The K-CVLT, following the same logic of the original CVLT ${ }^{30}$ that is a well-established measure of verbal memory, consists of newly selected stimulus items based on Korean language and culture. It assesses memory for a list of 16 items in 4 categories over 5 trials, Short- and Long-Delayed Free and Cued Recall. Because a word in the list is one of semantically related exemplars, the extent to which subjects use semantic strategies to recall items can be assessed as Semantic Clustering. For the assess- 
Table 1. Demographic and clinical characteristics of subjects

\begin{tabular}{|c|c|c|c|c|}
\hline & $\mathrm{BD} I(\mathrm{~N}=37)$ & BD II (N=46) & Controls $(\mathrm{N}=42)$ & Statistics \\
\hline Age & $32.1 \pm 9.0$ & $36.8 \pm 11.2$ & $34.1 \pm 7.4$ & $\mathrm{~F}=2.589, \mathrm{p}=0.079$ \\
\hline Female (\%) & $20(54.1)$ & $31(67.4)$ & $31(73.8)$ & $\chi^{2}=3.506, p=0.173$ \\
\hline Education years & $15.1 \pm 1.7$ & $14.8 \pm 2.0$ & $15.2 \pm 1.8$ & $\mathrm{~F}=0.498, \mathrm{p}=0.609$ \\
\hline Estimated IQ & $108.1 \pm 12.3$ & $111.2 \pm 14.2$ & $119.6 \pm 12.5$ & $\mathrm{~F}=8.296, \mathrm{p}<0.001$ \\
\hline Age at onset & $22.1 \pm 7.6$ & $24.9 \pm 9.9$ & & $\mathrm{~F}=2.125, \mathrm{p}=0.149$ \\
\hline Duration of illness & $10.1 \pm 6.2$ & $11.7 \pm 7.9$ & & $\mathrm{~F}=1.066, \mathrm{p}=0.305$ \\
\hline \multicolumn{5}{|l|}{ CGI-BP severity } \\
\hline Mania & $1.5 \pm 0.8$ & $1.4 \pm 0.7$ & & $\mathrm{~F}=2.230, \mathrm{p}=0.139$ \\
\hline Depression & $2.4 \pm 1.1$ & $2.7 \pm 1.1$ & & $\mathrm{~F}=0.976, \mathrm{p}=0.326$ \\
\hline Overall & $2.5 \pm 1.2$ & $2.6 \pm 1.1$ & & $\mathrm{~F}=0.319, \mathrm{p}=0.574$ \\
\hline Current mood states, $\mathrm{N}(\%)$ & & & & $\chi^{2}=5.502, p=0.064$ \\
\hline Euthymia & $24(64.9)$ & $26(56.6)$ & & \\
\hline Depressive episode & $10(27.0)$ & $20(43.5)$ & & \\
\hline (Hypo)manic episode & $0(0.0)$ & $0(0)$ & & \\
\hline Mixed episode & $3(8.1)$ & $0(0)$ & & \\
\hline \multicolumn{5}{|l|}{ Comorbidity, N (\%) } \\
\hline Any & $10(27.0)$ & $19(41.3)$ & & $\chi^{2}=1.537, p=0.181$ \\
\hline Anxiety disorders & $8(21.6)$ & $15(32.6)$ & & $\chi^{2}=1.236, p=0.194$ \\
\hline Substance use disorders & $4(10.8)$ & $6(13.0)$ & & $\chi^{2}=0.096, p=0.515$ \\
\hline Eating disorders & $1(2.7)$ & $6(13.0)$ & & $\chi^{2}=2.839, p=0.096$ \\
\hline
\end{tabular}

BD I: bipolar I disorder, BD II: bipolar II disorder, CGI-BP: Clinical Global Impression for Bipolar Disorder

ment of Semantic Clustering, subjects receive points based on the number of words they recall from the same category in succession. Score of 1 for five learning trials means a Clustering level that occurs by chance, and higher scores indicate more Semantic Clustering.

The Rey-Osterrieth Complex Figure Test (ROCF) $)^{31,32}$ was administered to all subjects. It consists of Copy, Immediate Recall, and 20-min Delayed Recall of a complex figure. The ROCF performances were scored by two clinical psychologists, using the Boston Qualitative Scoring System (BQSS). ${ }^{33}$ Among measures of executive aspects of visuoconstruction, the Organization score was used in this study.

The IQ was estimated with the Vocabulary and Block Design subtests from the Wechsler Adult Intelligence Scale-Revised (WAIS-R). ${ }^{34}$

\section{Statistics}

Group differences in demographic and clinical variables were examined using analysis of variance (ANOVA) and Pearson's chi-square tests. To explore the overall group differences in measures of two memory tasks before performing multiple subsequent tests for each of measures, one-way multivariate analysis of variance (MANOVA) was performed, with 13 memory measures as dependent variables and the group as a fac- tor. Each measure of the K-CVLT and the ROCF was compared between groups using one-way ANOVA, and post-hoc group comparisons were performed using the least square difference (LSD) test.

The relationships of demographic and clinical variables to memory performances were investigated using Pearson correlations. To examine if the mood states were associated with memory performances, we compared memory measures between patients in an euthymic state and patients in a depressive episode using ANOVA.

To examine the mediating effect of strategies on delayed recall, direct and mediated models were compared separately for the BD I and BD II group using a multiple regression analysis (Figure 1). In the direct model, group differences in delayed recall are expressed directly, with only group (controls= 0 , patients $=1$ ) as an independent variable. On the other hand, in the mediated model, whether group differences in delayed recall are mediated by strategies (Semantic Clustering for verbal recall and Organization for visual recall) was tested by entering group and strategies as independent variables into the structural equation. To test applicability of these path analytic models, relations between independent variables, mediators and dependent variables were tested using Pearson correlations. When relations of these variables were significant, the 


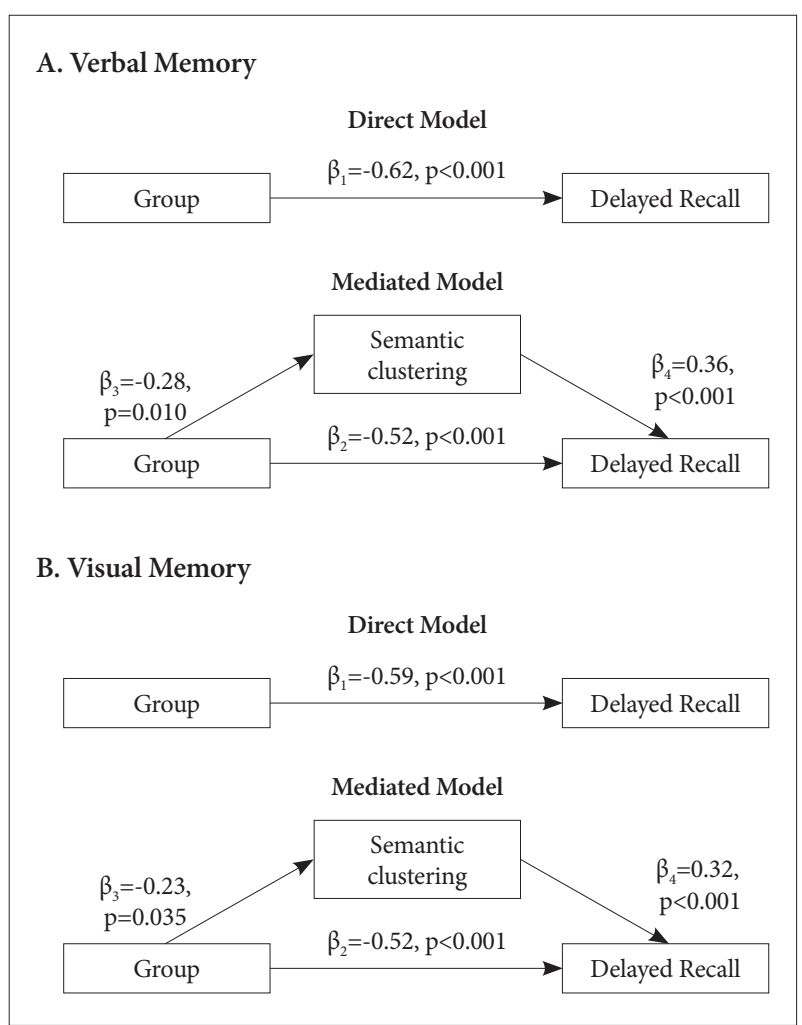

Figure 1. Direct and mediated model. $\beta$ value represents standardized regression coefficient. Note that $\beta_{2}$ decreased when inserting a mediator into the model. A: Two alternative path analytic models explaining group differences between patients with bipolar I disorder and controls in delayed recall in the Korean-California Verbal Learning Test. $R^{2}=0.38$ for the direct model and $R^{2}=$ 0.50 for the mediated model. B: Two alternative path analytic models explaining group differences between patients with bipolar I disorder and controls in delayed recall in the Rey-Osterrieth Complex Figure Test. $R^{2}=0.35$ for the direct model and $R^{2}=0.44$ for the mediated model.

path analytic model was applied.

The level of significance was set at $\mathrm{p}<0.05$ for all statistics.

\section{RESULTS}

\section{Performances on memory tasks}

The MANOVA revealed a significant main effect of group on memory performances $(\mathrm{F}=2.856, \mathrm{p}<0.001)$. The ANOVAs with LSD tests showed significant group differences in all measures of the K-CVLT and in Immediate Recall and Delayed Recall of the ROCF. The patients with BD I showed poorer performances on all measures of the K-CVLT and ROCF except Copy Accuracy of the ROCF than healthy controls. Effect sizes (Cohen's $\mathrm{d}$ ) of all verbal and visual recall scores of patients with BD I ranged from 1.00 to 1.53 that is regarded as large effects. However, the effect sizes for strategies were medium; 0.58 for Semantic Clustering and 0.47 for Organization score (Table 2). Patients with BD II performed poorly on both memory tasks as well. The mean scores of the ROCF
Copy Accuracy and Organization were not different between the BD II and control groups. The effect sizes of recall scores of the $\mathrm{BD}$ II group ranged from 0.44 to 0.86 that is generally regarded as medium effects (Table 2).

Although the patients with BD II performed more poorly than the controls, they were superior on all memory measures compared to the patients with BD I. The effect sizes for the differences between the $\mathrm{BD}$ II and $\mathrm{BD}$ I groups ranged from 0.41 to 0.77 (Table 2). However, there were no differences in the Semantic Clustering and Organization Score between the two patients groups.

Because the estimated IQ was different between the groups, we performed an additional ANCOVA with the IQ as a covariate. The differences in K-CVLT Long-Delayed Free Recall remained still significant $(\mathrm{F}=15.968, \mathrm{p}<0.001)$ after controlling for the IQ effects. The BD I group performed more poorly than the controls $(\mathrm{p}<0.001)$ and the BD II group $(\mathrm{p}=0.007)$. The $\mathrm{BD}$ II group performed more poorly than the controls $(\mathrm{p}=0.001$ ). Except the difference in ROCF Delayed Recall between the $\mathrm{BD}$ II group and the controls $(\mathrm{p}=0.140$ ), group differences in all other measures of verbal and visual recall remained significant after introducing the IQ as a covariate, the pattern of which was almost same to Table 2. Interestingly, however, group differences in the K-CVLT Semantic Clustering and the ROCF Organization Score did not reach the statistical significance after controlling for the IQ effects.

\section{Clinical correlations of memory performances}

Because our main interest was the delayed recall and strategies, we performed correlational analysis between these measures and clinical variables. Age and estimated IQ was not correlated to any measure of delayed recall and strategies in all three groups. In patients with BD I, CGI mania score was significantly correlated to Long-Delayed Free Recall of the KCVLT ( $r=-0.441, p=0.006)$, and age at onset was significantly correlated with Delayed Recall of the ROCF ( $r=-0.453$, $\mathrm{p}=$ 0.005). In patients with $\mathrm{BD}$ II, none of illness variables was related to any of memory performances. To explore the relationship of mood states to performances, we compared measures of the two memory tasks between euthymic patients and depressive patients. None of measures was different between patients in a depressive episode and euthymic patients. Compared to controls, euthymic patients in both patients groups performed poorly in all memory measures and in the K-CVLT Semantic Clustering. However, their performances on the ROCF Organization did not differ from those of controls $(\mathrm{F}=$ 1.994, $\mathrm{p}=0.142$ ).

\section{Multiple regression analyses}

In a series of correlational analyses to test applicability of 


\begin{tabular}{|c|c|c|c|c|c|c|c|}
\hline & $\begin{array}{c}\text { BD I } \\
(\mathrm{N}=37)\end{array}$ & $\begin{array}{c}\text { BD II } \\
(\mathrm{N}=46)\end{array}$ & $\begin{array}{l}\text { Controls } \\
(\mathrm{N}=42)\end{array}$ & Statistics & $\begin{array}{c}\text { Cohen's d } \\
\text { (BD I< } \\
\text { CTL) }\end{array}$ & $\begin{array}{c}\text { Cohen's d } \\
\text { (BD II< } \\
\text { CTL) }\end{array}$ & $\begin{array}{c}\text { Cohen's d } \\
(\text { BD I< } \\
\text { BD II) }\end{array}$ \\
\hline \multicolumn{8}{|l|}{ K-CVLT } \\
\hline Trial 1 & $5.08 \pm 1.99$ & $6.28 \pm 2.08$ & $7.45 \pm 2.19$ & $\mathrm{~F}=12.635, \mathrm{p}<0.001$ & $1.13^{* *}$ & $0.55^{*}$ & $0.59^{*}$ \\
\hline Trial 5 & $10.35 \pm 3.40$ & $12.28 \pm 2.71$ & $13.62 \pm 1.98$ & $\mathrm{~F}=14.221, \mathrm{p}<0.001$ & $1.18^{* *}$ & $0.56^{*}$ & $0.63^{*}$ \\
\hline Trial B & $4.59 \pm 1.40$ & $5.37 \pm 2.21$ & $6.29 \pm 1.93$ & $\mathrm{~F}=7.822, \mathrm{p}=0.001$ & $1.00^{* *}$ & $0.44^{*}$ & 0.42 \\
\hline Total Score & $42.14 \pm 12.42$ & $48.98 \pm 10.98$ & $57.00 \pm 7.55$ & $\mathrm{~F}=20.065, \mathrm{p}<0.001$ & $1.45^{* *}$ & $0.85^{* *}$ & $0.58^{*}$ \\
\hline Short-Delayed Free Recall & $9.14 \pm 3.81$ & $11.04 \pm 2.99$ & $12.83 \pm 2.28$ & $\mathrm{~F}=14.350, \mathrm{p}<0.001$ & $1.17^{* *}$ & $0.67^{*}$ & $0.56^{*}$ \\
\hline Short-Delayed Cued Recall & $10.08 \pm 3.03$ & $11.41 \pm 2.65$ & $13.33 \pm 2.41$ & $\mathrm{~F}=14.686, \mathrm{p}<0.001$ & $1.19^{* *}$ & $0.76^{*}$ & $0.47^{*}$ \\
\hline Long-Delayed Free Recall & $9.43 \pm 3.22$ & $11.28 \pm 3.24$ & $13.62 \pm 2.15$ & $F=20.576, p<0.001$ & $1.53^{* *}$ & $0.85^{* *}$ & $0.57^{*}$ \\
\hline Long-Delayed Cued Recall & $10.49 \pm 3.08$ & $11.72 \pm 2.93$ & $13.86 \pm 1.92$ & $\mathrm{~F}=16.131, \mathrm{p}<0.001$ & $1.31^{* *}$ & $0.86^{* *}$ & $0.41^{*}$ \\
\hline Semantic Clustering & $1.94 \pm 0.70$ & $2.10 \pm 0.75$ & $2.38 \pm 0.81$ & $\mathrm{~F}=4.571, \mathrm{p}=0.012$ & $0.58^{*}$ & $0.54^{*}$ & 0.05 \\
\hline \multicolumn{8}{|l|}{ ROCF } \\
\hline Copy Accuracy & $32.58 \pm 4.82$ & $32.84 \pm 4.02$ & $33.58 \pm 2.25$ & $\mathrm{~F}=0.819, \mathrm{p}=0.443$ & 0.28 & 0.23 & 0.06 \\
\hline Immediate Recall & $14.50 \pm 7.84$ & $18.64 \pm 7.63$ & $23.08 \pm 5.28$ & $\mathrm{~F}=14.849, \mathrm{p}<0.001$ & $1.28^{* *}$ & $0.68^{*}$ & $0.54^{*}$ \\
\hline Delayed Recall & $13.59 \pm 6.88$ & $19.00 \pm 7.08$ & $22.19 \pm 4.97$ & $\mathrm{~F}=18.019, \mathrm{p}<0.001$ & $1.34^{* *}$ & $0.52^{*}$ & $0.77^{* *}$ \\
\hline Organization Score & $5.68 \pm 1.81$ & $5.80 \pm 1.81$ & $6.45 \pm 1.49$ & $\mathrm{~F}=2.438, \mathrm{p}=0.092$ & $0.47^{*}$ & 0.39 & 0.07 \\
\hline
\end{tabular}

${ }^{*} \mathrm{p}<0.05$ after the LSD (least square difference method) post-hoc comparison to controls, ${ }^{* *} \mathrm{p}<0.001$ after the LSD post-hoc comparison to controls. BD I: bipolar I disorder, BD II: bipolar II disorder, CTL: controls, K-CVLT: Korean-California Verbal Learning Test, ROCF: ReyOsterrieth Complex Figure Test

the path analytic models for the BD I group, the group (BD I and control) was significantly correlated with the K-CVLT Semantic Clustering ( $r=0.280, p=0.013)$, K-CVLT Long-Delayed Free Recall $(\mathrm{r}=0.616, \mathrm{p}<0.001)$, ROCF Organization $(\mathrm{r}=0.232$, $\mathrm{p}=0.040)$, and the ROCF Delayed Recall $(\mathrm{r}=0.590, \mathrm{p}<0.001)$. In the BD I group, the K-CVLT Semantic Clustering was significantly correlated with the K-CVLT Long-Delayed Recall $(\mathrm{r}=0.388, \mathrm{p}=0.018)$, and the ROCF Organization was significantly correlated with the ROCF Delayed Recall ( $r=0.371$, $\mathrm{p}=$ 0.024). In controls, the K-CVLT Semantic Clustering was significantly correlated with the K-CVLT Long-Delayed Recall $(\mathrm{r}=0.491, \mathrm{p}=0.001)$, and the ROCF Organization was significantly correlated with the ROCF Delayed Recall ( $\mathrm{r}=0.345$, $\mathrm{p}=$ 0.025). For the BD II group, the group (BD II and control) was significantly correlated with the K-CVLT Semantic Clustering $(r=0.252, p=0.018)$, K-CVLT Long-Delayed Free Recall $(\mathrm{r}=0.401, \mathrm{p}<0.001)$, and the ROCF Delayed Recall $(\mathrm{r}=0.220$, $\mathrm{p}=0.040$ ). However, the group did not related to the ROCF Organization $(\mathrm{r}=0.180, \mathrm{p}=0.090)$. In the $\mathrm{BD}$ II group, the $\mathrm{K}$ CVLT Semantic Clustering was significantly correlated with the Long-Delayed Recall $(\mathrm{r}=0.379, \mathrm{p}=0.006)$, and the ROCF Organization was significantly correlated with the ROCF Delayed Recall $(\mathrm{r}=0.488, \mathrm{p}=0.001)$. Because the prerequisite for path analysis of the ROCF measures in the BD II group had not been fulfilled, path analysis for visual memory was performed only for the BD I group.

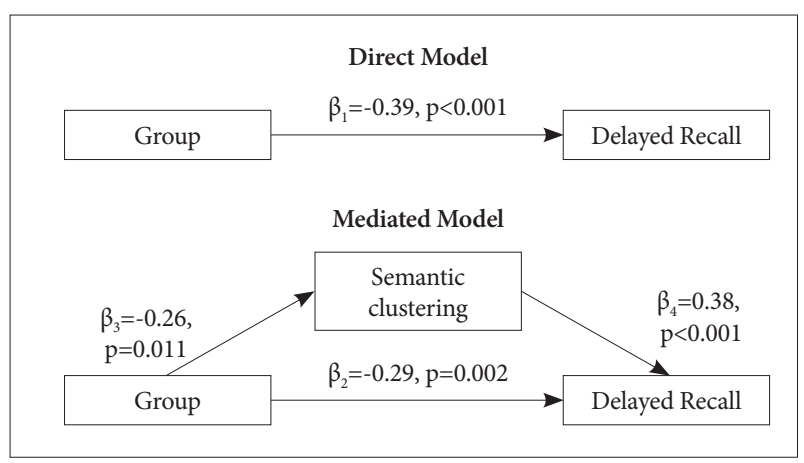

Figure 2. Two alternative path analytic models explaining group differences between patients with bipolar II disorder and controls in delayed recall in the Korean-California Verbal Learning Test. $\mathrm{R}^{2}=0.15$ for the direct model and $\mathrm{R}^{2}=0.29$ for the mediated model.

In the direct model for verbal memory in the BD I group, the model accounted for $38 \%$ of the variance of K-CVLT LongDelayed Free Recall. On the other hand, the mediated model accounted for $50 \%$ of variance of the verbal recall. For visual memory in the BD I group, the direct model accounted for $35 \%$ of the variance of ROCF Delayed Recall and the mediated model for $44 \%$ of the variance of the visual recall (Figure 1). In the BD II group, the direct model for verbal memory accounted for $15 \%$ of the variance of the K-CVLT Long-Delayed Free Recall which was increased to $29 \%$ when the mediated model was applied (Figure 2). In three mediated models, group differences in delayed recall remained significant. 
We re-applied the path analytic models for euthymic patients (24 patients with BD I, 26 patients with BD II and 42 controls) to test if the partial mediating effects of strategies are also found in euthymic state. Because the ROCF Organization Scores were not related to the groups, the model was applied only to verbal memory. The results were almost same to the findings that were yielded from all subjects. In the BD I group, the direct model accounted for $33 \%$ of the variance of K-CVLT Long-Delayed Free Recall and the mediated model for $46 \%$ of the recall $\left(\beta_{1}=-0.58, p<0.001 ; \beta_{2}=-0.49, p<0.001\right.$; $\left.\beta_{3}=-0.25, p=0.010 ; \beta_{4}=-0.36, p<0.001\right)$. In the BD II group, the direct model accounted for $13 \%$ and the mediated model for $30 \%$ of the recall $\left(\beta_{1}=-0.36, p=0.001 ; \beta_{2}=-0.25, p=0.019 ; \beta_{3}=\right.$ $\left.-0.27, \mathrm{p}=0.024 ; \beta_{4}=-0.43, \mathrm{p}<0.001\right)$.

\section{DISCUSSION}

The main finding of this study is that verbal and visual memory was impaired in both BD I and BD II groups and that deficits in organizational strategies had only a partial mediating effect on memory deficits in BD I and BD II. The deficit in verbal memory was partly mediated by difficulties in Semantic Clustering in both subtypes, whereas the mediating effect of difficulties in Organization on the visual memory deficit was present only in $\mathrm{BD}$ I. To our knowledge, this is the first study to explore the relationships between memory performances and strategies during encoding in BD II.

The profile of performances in BD I in this study was consistent with previous reports. ${ }^{1}$ Regardless of the information modality, the effect sizes of impairments in delayed recall were large in BD I. However, the effect sizes of Semantic Clustering and Organization were medium. Although the mediating effects of deficits in these strategies were significant, differences in verbal and visual recall between the BD I and control groups remained still significant. These findings are consistent with a previous study ${ }^{7}$ that has reported a weaker mediating effect of difficulties in Semantic Clustering between BD I patients and controls than that between OCD patients and controls. This suggests that patients with BD I have verbal memory problems that are not fully attributable to a failure in organizational strategies. Regarding visual memory impairment, our results showed similar findings to verbal memory impairment. Deckersbach et al. ${ }^{13}$ however, has reported that differences in ROCF Delayed Free Recall between BD I patients and controls did not remain significant when effects of lower organization were partialled out, which is inconsistent with our findings on visual memory impairment. One possible reason for these different findings may be explained by the sample characteristics. The patients in their study, compared to our sample, had an older age (39.9 vs. 32.1 years), earlier age at on- set (16.9 vs. 22.1 years) and longer duration of illness (22.6 years vs. 10.1 years), and were euthymic. Progression of illness or a subtype with an earlier onset might have potentiated deficits in executive functions and caused increased mediating effects of executive dysfunctions on visual memory impairment. In their additional analyses, the number of mood episodes was found to be negatively correlated with Organization, which supports this tentative hypothesis. In our results, onset age was negatively correlated to ROCF Delayed Recall as well. Nevertheless, our data have a strength over previous ones in that measures of the K-CVLT and ROCF were obtained from the same sample and yielded results of similar pattern. Whether deficits in organization strategies for different information modalities would differently mediate memory impairment remains as an open question and needs further research.

The performances on verbal memory in patients with BD II and their medium effect sizes are quite consistent with previous reports. ${ }^{21,23,24,35}$ Among 5 studies that investigated verbal memory functions in BD II, only one study ${ }^{22}$ has reported negative findings. Meanwhile, visual memory in BD II was explored in a previous study $y^{21}$ in which the ROCF performance was impaired as well. Taken together, memory impairments with medium effect sizes are evident in BD II. However, none of previous studies on BD II presented organizational strategies, making it difficult to compare our results to previous ones. In the current study, K-CVLT Semantic Clustering was lower in patients with BD II compared to controls, whereas ROCF Organization was not impaired in BD II. In a multiple regression analysis for verbal memory impairment in BD II, difficulties in Semantic Clustering partly mediated the impairment of free recall, and differences in Long-Delayed Free Recall between patients and controls remained still significant in a mediated model. This pattern is exactly similar to the case of BD I. Therefore, our results suggest that verbal memory impairment may be one of the fundamental cognitive deficits in bipolar disorders and that executive dysfunctions can exert an additional influence on memory impairments. The extent to which executive dysfunctions may have an additional effect on memory performances may differ across subtypes of bipolar illness, clinical characteristics and information modalities. One of such an example may be the absence of mediating effects of difficulties in ROCF Organization in patients with BD II on ROCF delayed recall impairment for visual information. To explore these subtle effects in BD II, we may need a larger or well-controlled sample.

Torres et al. ${ }^{2}$ found core cognitive deficits that were present at illness onset and suggested that these deficits were not exclusively attributable to illness progression, which also supports our hypothesis that memory impairments are a fundamental deficit in bipolar disorder. In their comparison of the 
magnitude of cognitive impairments in first-episode patients with that of previously published multi-episode patients, deficits in executive function and verbal memory were smaller in first-episode. ${ }^{2}$ Therefore, deficits in memory and executive functions may worsen further with advancing illness course, possibly due to the toxic effects of recurrent episodes or oxidative stress on brain. We suggest that those memory and executive impairments may worsen reciprocally in advances stages of illness and the role or degree of executive impairments may vary across the clinical spectrum.

Generally, effect sizes of memory impairments in BD I were larger than those in BD II, which is consistent with our prior hypotheses. Furthermore, the percent of variance for which a path analytic model accounted was larger in BD I than in $\mathrm{BD}$ II. Whether this different pattern is associated with the involvement of distinct neural networks remains elusive. In future research, we need to investigate whether the differences in cognitive and neurobiological measures between BD I and BD II are quantitative or qualitative and how they relate to each others to deepen understanding the pathophysiology of bipolar disorders.

This study has some limitations. First, the three groups were not completely matched for demographic and clinical variables. Particularly, the estimated IQ was different among the groups, which might have exerted a potential confounding effect on group differences in memory measures. However, in additional analyses, the IQ was not correlated with any of memory performances in each of the three groups, and thus could not mediate the group differences in memory measures. And we found very similar group differences in memory performances when introducing the IQ as a covariate. Because we estimated IQ by a short-version of the WAIS-R, the group differences in IQ might have been inaccurately exaggerated. Second, all subjects except two were taking medications and patients with BD I were taking lithium or antipsychotics more frequently than patients BD II. Although the daily doses of each medication were not different between two patients groups, potential medication effects on cognitive functions could not be excluded. Third, the sample consisted of heterogeneous patients with regard to clinical features. Approximately one third of patients were in a depressive episode and two thirds were euthymic. However, the ratio of depressives was not different between the two patients groups, and host-hoc comparisons did not reveal any differences in memory measures between patients in a depressive episode and euthymic patients in each group. Furthermore, group comparisons of only euthymic patients revealed the same profile of cognitive impairments and the same relationship of memory and organization strategies. Therefore, different mood states of our sample could not weaken our findings. Patients with psychiatric comorbid conditions were not excluded. Again, these comorbidity did not have an effect on memory measures in each group and on group differences in post-hoc analyses. Regarding chronicity and comorbidity, we need further investigation using a more homogeneous sample. Third, clinical symptoms and their severities that had been measured using ratings scales were not available in a significant proportion of subjects, and we stratified mood states according to clinical interview and CGI-BP score. Despite these limitations, the present study, using a decent number of subjects, showed memory impairments in bipolar disorders that were not fully attributable to poor organizational strategies. Strengths of this study include concurrent investigation of verbal and visual memory performances from the same sample and a high generalizability due to the broad inclusion criteria.

In conclusion, verbal and visual memory impairments were evident not only in BD I but also in BD II. Impaired recall of verbal materials in bipolar disorders was influenced by but not fully mediated by organizational strategies.

\section{Acknowledgments}

This study was supported by a grant A101915 from the Korea Healthcare Technology R\&D Projects, Ministry of Health \& Welfare, Republic of Korea.

\section{REFERENCES}

1. Robinson LJ, Thompson JM, Gallagher P, Goswami U, Young AH, Ferrier IN, et al. A meta-analysis of cognitive deficits in euthymic patients with bipolar disorder. J Affect Disord 2006;93:105-115.

2. Torres IJ, DeFreitas VG, DeFreitas CM, Kauer-Sant'Anna M, Bond DJ, Honer WG, et al. Neurocognitive functioning in patients with bipolar I disorder recently recovered from a first manic episode. J Clin Psychiatry 2010;71:1234-1242.

3. Bora E, Yucel M, Pantelis C. Cognitive endophenotypes of bipolar disorder: a meta-analysis of neuropsychological deficits in euthymic patients and their first-degree relatives. J Affect Disord 2009;113:1-20.

4. Savage CR. Neuropsychology of subcortical dementias. Psychiatr Clin North Am 1997;20:911-931.

5. Deckersbach T, Otto MW, Savage CR, Baer L, Jenike MA. The relationship between semantic organization and memory in obsessive-compulsive disorder. Psychother Psychosom 2000;69:101-107.

6. Penades R, Catalan R, Andres S, Salamero M, Gasto C. Executive function and nonverbal memory in obsessive-compulsive disorder. Psychiatry Res 2005;133:81-90.

7. Deckersbach T, Savage CR, Reilly-Harrington N, Clark L, Sachs G, Rauch SL. Episodic memory impairment in bipolar disorder and obsessive-compulsive disorder: the role of memory strategies. Bipolar Disord 2004;6:233-244.

8. Shin MS, Park SJ, Kim MS, Lee YH, Ha TH, Kwon JS. Deficits of organizational strategy and visual memory in obsessive-compulsive disorder. Neuropsychology 2004;18:665-672.

9. Brebion G, David AS, Jones H, Pilowsky LS. Semantic organization and verbal memory efficiency in patients with schizophrenia. Neuropsychology 2004;18:378-383.

10. Bora E, Fornito A, Yucel M, Pantelis C. Voxelwise meta-analysis of gray matter abnormalities in bipolar disorder. Biol Psychiatry 2010;67:10971105.

11. Chen $\mathrm{CH}$, Suckling J, Lennox BR, Ooi C, Bullmore ET. A quantitative meta-analysis of fMRI studies in bipolar disorder. Bipolar Disorder 
2011;13:1-15.

12. Bearden CE, Glahn DC, Monkul ES, Barrett J, Najt P, Kaur S, et al. Sources of declarative memory impairment in bipolar disorder: mnemonic processes and clinical features. J Psychiatr Res 2006;40:47-58.

13. Deckersbach T, McMurrich S, Ogutha J, Savage CR, Sachs G, Rauch SL. Characteristics of non-verbal memory impairment in bipolar disorder: the role of encoding strategies. Psychol Med 2004;34:823-832.

14. Judd LL, Akiskal HS, Schettler PJ, Coryell W, Endicott J, Maser JD, et al. A prospective investigation of the natural history of the long-term weekly symptomatic status of bipolar II disorder. Arch Gen Psychiatry 2003;60:261-269.

15. Merikangas KR, Akiskal HS, Angst J, Greenberg PE, Hirschfeld RM, Petukhova M, et al. Lifetime and 12-month prevalence of bipolar spectrum disorder in the National Comorbidity Survey replication. Arch Gen Psychiatry 2007;64:543-552.

16. Maina G, Albert U, Bellodi L, Colombo C, Faravelli C, Monteleone P, et al. Health-related quality of life in euthymic bipolar disorder patients: differences between bipolar I and II subtypes. J Clin Psychiatry 2007; 68:207-212.

17. Heun R, Maier W. The distinction of bipolar II disorder from bipolar I and recurrent unipolar depression: results of a controlled family study. Acta Psychiatr Scand 1993;87:279-284.

18. McMahon FJ, Simpson SG, McInnis MG, Badner JA, MacKinnon DF, DePaulo JR. Linkage of bipolar disorder to chromosome 18q and the validity of bipolar II disorder. Arch Gen Psychiatry 2001;58:1025-1031.

19. Ha TH, Her JY, Kim JH, Chang JS, Cho HS, Ha K. Similarities and differences of white matter connectivity and water diffusivity in bipolar I and II disorder. Neurosci Lett 2011;505:150-154.

20. Ha TH, Ha K, Kim JH, Choi JE. Regional brain gray matter abnormalities in patients with bipolar II disorder: a comparison study with bipolar I patients and healthy controls. Neurosci Lett 2009;456:44-48.

21. Andersson S, Barder HE, Hellvin T, Lovdahl H, Malt UF. Neuropsychological and electrophysiological indices of neurocognitive dysfunction in bipolar II disorder. Bipolar Disord 2008;10:888-899.

22. Simonsen C, Sundet K, Vaskinn A, Birkenaes AB, Engh JA, Hansen CF, et al. Neurocognitive profiles in bipolar I and bipolar II disorder: differences in pattern and magnitude of dysfunction. Bipolar Disord 2008; 10:245-255.
23. Sole B, Bonnin CM, Torrent C, Balanza-Martinez V, Tabares-Seisdedos R, Popovic D, et al. Neurocognitive impairment and psychosocial functioning in bipolar II disorder. Acta Psychiatr Scand 2012;125:309-317.

24. Torrent C, Martinez-Aran A, Daban C, Sanchez-Moreno J, Comes M, Goikolea JM, et al. Cognitive impairment in bipolar II disorder. Br J Psychiatry 2006;189:254-259.

25. Chang JS, Choi S, Ha K, Ha TH, Cho HS, Choi JE, et al. Differential pattern of semantic memory organization between bipolar I and II disorders. Prog Neuropsychopharmacol Biol Psychiatry 2011;35:1053-1058.

26. First MB, Spitzer RL, Gibbon M, Williams JB. Structured Clinical Interview for DSM-IV Axis I Disorder. New York: New York State Psychiatric Institute; 1996.

27. Sheehan DV, Lecrubier Y, Sheehan KH, Amorim P, Janavs J, Weiller E, et al. The Mini-International Neuropsychiatric Interview (M.I.N.I.): the development and validation of a structured diagnostic psychiatric interview for DSM-IV and ICD-10. J Clin Psychiatry 1998;59(Suppl 20): 22-33.

28. Spearing MK, Post RM, Leverich GS, Brandt D, Nolen W. Modification of the Clinical Global Impressions (CGI) Scale for use in bipolar illness (BP): the CGI-BP. Psychiatry Res 1997;73:159-171.

29. Kim JK, Kang Y. Normative study of the Korean-California Verbal Learning Test (K-CVLT). Clin Neuropsychol 1999;13:365-369.

30. Delis DC, Kramer JH, Kaplan E, Ober BA. California Verbal Learning Test Research Edition Manual Adult Version. San Antonio: The Psychological Corporation; 1987.

31. Rey A. L'examen psychologique dans les cas d'encephalopathie traumatique. Arch Psychol 1941;28:286-340.

32. Osterrieth PA. Le test de copie d'une figure complexe. Arch Psychol 1944;30:206-356.

33. Stern RA, Singer EA, Duke LM, Singer NG, Morey CE, Daughtrey EW, et al. The Boston Qualitative Scoring System for the Rey-Osterrieth Complex Figure: Description and interrator reliability. Clin Neuropsychol 1994;8:309-322.

34. Weschsler D. Adult Intelligence Scale, Revised (WAIS-R) Manual. San Antonio: Psychological Corp; 1981.

35. Martino DJ, Igoa A, Marengo E, Scapola M, Strejilevich SA. Neurocognitive impairments and their relationship with psychosocial functioning in euthymic bipolar II disorder. J Nerv Ment Dis 2011;199:459-464. 